\title{
In vino veritas - quo vadis, publicitas?
}

Quel rapport y a-t-il entre un verre de bon vin que l'on déguste, par exemple un Bordeaux, un Chardonnay, un Riesling ou un Chianti, et une vessie hyperactive? Anna Ebel peut vous l'expliquer. Vous ne connaissez pas Madame Ebel? C'est la chroniqueuse sympathique et souriante de la rubrique Info Santé du Migros Magazine, qui donne à son lectorat bienveillant (lequel compte, relevons-le, pas moins de trois millions de personnes) des conseils utiles pour une vie saine et active: «Il n'y a pas de bon repas sans un petit verre de vin - automne ou pas», dit-elle, ce qui apparemment ne pose pas de problème puisque «Boire du vin avec modération peut être bon pour la santé». Ouf, pense-t-on, il n'y a rien de faux dans mes habitudes.

Mais attention, tout n'est pas si simple, ce serait trop beau. Les explications de Mme Ebel prennent une tournure plus didactique: «La prudence s'impose toutefois pour les personnes qui ont une vessie hyperactive», relève-t-elle. Une digression bien intentionnée s'ensuit, qui vise à familiariser le public - peut-être un peu perplexe - avec les symptômes de ce trouble de santé. Le tout se termine par un conseil destiné aux personnes qui en sont atteintes: «Il est conseillé d'aller consulter son médecin si l'on veut conserver sa qualité de vie.»

«In vino veritas», tel est le titre intelligemment donné par Mme Ebel à son «Info Santé». On ne trouvera ma foi guère d'arguments à opposer à une rubrique journalistique sur les questions de santé: ce sont des thèmes qui intéressent les gens. Là où le bât blesse, c'est que nous avons affaire dans ce cas à une annonce publicitaire, mais qui se fond parfaitement dans la mise en page de la partie rédactionnelle du magazine. Si ce fait est quand même - signalé dans l'édition française par le mot «Publicité» imprimé en petits caractères tout en haut de la chronique, l'édition allemande n'en pipe pas mot. Tout au plus les lecteurs capables de déchiffrer un microfilm sans lunettes découvrent-ils, en bas de page, la mention discrète d'une grande entreprise pharmaceutique et d'une agence de relations publiques. Inutile d'en rajouter, on aura compris de quoi il retourne.

Voulons-nous lancer ici une bordée de critiques contre l'industrie pharmaceutique, dans une revue qui, rappelons-le, se finance substantiellement par des annonces émanant de cette branche? Certes, un tel procédé serait probablement possible sans «retour de bâton», ce qui confirmerait que l'indépendance rédactionnelle du BMS, en tout temps essentielle pour les Editions médicales suisses, est respectée par les annonceurs. Mais une attaque de ce genre n'irait pas dans le sens de notre propos. Les métaphores guerrières ne sont guère aptes à décrire les relations entre corps médical, presse (spécialisée) et industrie, il faut leur préférer un langage de partenariat. En fin de compte, ce sont les entreprises pharmaceutiques qui livrent aux praticiens les instruments indispensables à leur activité médicale et à la médecine moderne.

La publicité fait partie des réalités économiques les plus largement acceptées dans notre société dite libre. Et lorsqu'il s'agit de promouvoir des médicaments, des règles particulières sont applicables. Il est évident que pour les stratèges du marketing, dans un environnement qui se durcit, un jonglage habile avec ces règles peut devenir une forme de défi concurrentiel. Après tout, des gains élevés sont en jeu, il en va de millions à gagner.

Mais tous les moyens sont-ils bons pour atteindre cet objectif? S'agissant de maladie, de santé et de patients, on parle souvent de confiance mutuelle, de respect, d'honnêteté et de dignité. Est-ce instaurer la confiance, est-ce faire montre de respect et d'honnêteté que de leurrer pour plus ou moins systématiquement les patients (potentiels) - de même que les médecins - en recourant à des publireportages et à d'autres formes de publicité semblables?

Souhaiter davantage de transparence dans la publicité relève d'une vision naïve des choses, pourrait-on penser. Cependant, des indices toujours plus nombreux indiquent que bien des collègues partagent cette façon de voir. A témoin, par exemple, les quelques lettres de lecteurs que nous publions dans le présent numéro. Ce n'est pas la raison d'être de la publicité qui est remise en cause, mais le fait que celle-ci, en quelque sorte, se désavoue en épousant les formes d'une information journalistique.

$\mathrm{Au}$ vu de ces courriers abordant la question des publireportages**, nous avons demandé à Jean Martin, ancien médecin cantonal vaudois, d'approfondir cette thématique dans un article. Vous lirez le résultat - à mon avis impressionnant - de son travail à la page 1600 de la présente édition. Il ne reste qu'à espérer que Mme Ebel lira elle aussi ces considérations et que peut-être même elle en tiendra compte.

Bruno Kesseli, rédacteur en chef
* Les directives de la FMH concer-
nant les publireportages ont été

publiées dans le $\mathrm{BMS} \mathrm{N}^{\circ}$ 20/2006.
No $35 / 2006$ du 28 août 2006 édition française p. 65 , édition allemande p. 85 . 